\title{
Molecular characterization of the cystic fibrosis transmembrane conductance regulator gene in congenital absence of the vas deferens
}

Ana Grangeia, $B S^{1}$, Rosália Sá, $B S^{2}$, Filipa Carvalho, $P h D^{1}$, Josiane Martin, $P h D^{3}$, Emmanuelle Girodon, $M D^{3}$, Joaquina Silva, $M D^{4}$, Luís Ferráz, $M D^{4}$, Alberto Barros, $M D, P h D^{1,4}$, Mário Sousa, $M D, P h D^{1,2,4}$

\begin{abstract}
Purpose: Approximately $20 \%$ of patients with congenital absence of the vas deferens remain without two mutations identified. We applied a strategy of serial screening steps to 45 patients with congenital absence of the vas deferens and characterized cystic fibrosis transmembrane conductance regulator gene mutations in all cases. Methods: DNA samples of 45 patients with congenital absence of the vas deferens were screened by successive different molecular genetics approaches. Results: Initial screening for the 31 most frequent cystic fibrosis mutations, IVS 8 poly $(\mathrm{TG}) \mathrm{m}$, poly(T)n, and M470V polymorphisms, identified 8 different mutations in 40 patients (88.9\%). Extensive cystic fibrosis transmembrane conductance regulator gene analysis by denaturing gradient gel electrophoresis, denaturing high-performance liquid chromatography, and DNA sequencing detected 17 further mutations, of which three were novel. Cystic fibrosis transmembrane conductance regulator gene rearrangements were searched by semiquantitative fluorescent multiplex polymerase chain reaction, which detected a CFTRdele2,3 (21 kb) large deletion and confirmed two homozygous mutations. Overall, 42 patients (93.3\%) had two mutations and 3 patients (6.7\%) had one mutation detected. Conclusions: The present screening strategy allowed a higher mutation detection rate than previous studies, with at least one cystic fibrosis transmembrane conductance regulator gene mutation found in all patients with congenital absence of the vas deferens. Genet Med 2007:9(3):163-172.
\end{abstract}

Key Words: CAVD, CBAVD, CUAVD, CFTR mutations, CFTR rearrangements, cystic fibrosis, male infertility

Congenital absence of the vas deferens (CAVD) in otherwise healthy males is responsible for $1 \%$ to $2 \%$ of male infertility and $6 \%$ of obstructive azoospermia cases. The disease was classified as a primary genital form of cystic fibrosis (CF) because $67 \%$ to $85 \%$ of patients with CAVD have mutations and/or splicing variants in the cystic fibrosis transmembrane conductance regulator (CFTR) gene. Patients with CAVD with upper urinary tract malformations ( $20 \%$ of cases) have a normal frequency of CFTR mutations and are thus considered a distinct clinical entity with a different cause. ${ }^{1-8}$

CFTR genotypes in patients with CAVD are markedly different from those found in patients with CF. Patients with CAVD are usually heterozygous for a mild mutation on one allele and a severe mutation on the second allele $(88 \%)$, or for mild mutations on both CFTR alleles (12\%). On the other

\footnotetext{
From the ${ }^{1}$ Department of Genetics, Faculty of Medicine, University of Porto, Portugal; ${ }^{2}$ Lab Cell Biology, ICBAS, University of Porto, Portugal; ${ }^{3}$ Department of Biochemistry and Genetics, Henri Mondor Hospital, Creteil, France; ${ }^{4}$ Centre for Reproductive Genetics A. Barros, Porto, Portugal.

Ana Grangeia, BS, Department of Genetics, Faculty of Medicine, University of Porto, Alameda Prof Hernâni Monteiro, 4200-319 Porto, Portugal.E-mail: anafg@med.up.pt

Submitted for publication October 10, 2006.

Accepted for publication December 12, 2006.

The authors declare no conflict of interest.
}

DOI: 10.1097/GIM.0b013e3180318aaf hand, homozygosity or compound heterozygosity for two severe mutations is responsible for the classic CF phenotype. The most common CFTR mutations found in CAVD are T5 allele, deltaF508, and $\mathrm{R} 117 \mathrm{H}$, with the combination between the T5 allele and a severe CF mutation in the other allele being the main cause of CAVD. ${ }^{9}$

The polymorphic polythymidine locus (Tn) is located within the $3^{\prime}$ splice acceptor site of intron 8 (IVS8 poly[T]n). The length of the IVS8 poly(T)n tract (T5/T7/T9) is inversely correlated with the degree of alternative splicing of exon 9. Because the protein product of the CFTR transcript lacking exon 9 is devoid of cyclic adenosine monophosphate-activated chloride conductance, the T5 allele is considered a mild mutation with incomplete penetrance. ${ }^{10-13}$ Intronic (TG)m and exonic (M470V) cis-acting elements additionally influence the alternative splicing of exon 9. The polymorphic IVS8 poly(TG)m tract consists of a variable number (9-13) of repeats and is located immediately upstream of the IVS8 poly(T)n tract. Because of self-base pairing and RNA hairpin formation, which interfere with splicing factor interactions and the recognition of the intron 8/exon 9 junction, longer (TG)m tracts (12/13 repeats) favor the penetrance of the IVS8(T) 5 allele and lead to a higher number of transcripts lacking exon 9. The M470V missense polymorphism is located in exon 10 and influences the penetrance of the T5 allele, because the V470 variant increases the proportion of mRNAs lacking exon 9 and thus contributes to even lower levels of normal transcripts. ${ }^{14-20}$ 
Screening for the most common CF mutations does not allow the diagnosis of a significant proportion of patients with CAVD. Analysis of the whole CFTR gene increases the mutation detection rate, but mutations still will not be detected in a subset of patients with CAVD. This could be attributed to the presence of intronic mutations, the contribution of another gene, or large CFTR gene rearrangements. ${ }^{21-25}$ In the present study, we determined the type and frequency of CFTR gene mutations/variations in 45 Portuguese patients with CAVD. Panel CF screening, whole CFTR gene screening by denaturing gradient gel electrophoresis (DGGE), denaturing high-performance liquid chromatography (dHPLC) and DNA sequencing, IVS8 poly(T)n analysis, (TG)m and M470V polymorphism evaluation, and CFTR gene rearrangement analysis by semiquantitative fluorescent multiplex polymerase chain reaction (QFM-PCR) enabled us to detect mutations in all cases (two mutations in $93 \%$ of the patients), with description of three novel mutations.

\section{MATERIALS AND METHODS}

\section{Patients}

Forty-five patients with CAVD were diagnosed during the period 1999 to 2004 (43 of Portuguese descent, 1 of Ukrainian descent, and 1 of Indian descent). Forty-two patients had congenital bilateral absence of the vas deferens (CBAVD), and three patients had congenital unilateral absence of the vas deferens (CUAVD). Patients had a good general condition with no clinical manifestations or family history suggestive of CF, including pancreatic insufficiency, other gastrointestinal symptoms, or chronic airways disease. Diagnosis was based on azoospermia with low seminal fluid volume $(<1.5 \mathrm{~mL}), \mathrm{pH}$ $(<7.2)$ and/or fructose levels, and physical examination of the scrotum (presence of globus major and absence of palpable vas deferens). Abdominal ultrasonography was performed to rule out renal abnormalities. Transrectal and scrotal ultrasonography showed hypoplasia/aplasia of the seminal vesicles and a dilated epididymis head. Patients had normal serum hormone levels and a normal 46, XY karyotype, and tested negative for Y-chromosome AZF and DAZ microdeletions. At the diagnostic testicle biopsy, all patients showed conserved spermatogenesis with germ cell sloughing, suggestive of tubular obstruction. Sperm retrieval for infertility treatment was performed by microsurgical epididymal sperm aspiration or open testicular sperm extraction, depending on the presence of enough motile sperm in the dilated epididymis. Absence of the vas deferens and of distal epididymis, and presence of epididymis globus major were confirmed during the surgical procedure. ${ }^{26}$ All patients were diagnosed and treated by a single experienced urologist. Genetic analyses were performed under patient-informed consent.

\section{CFTR mutation panel testing}

DNA samples were obtained from peripheral blood lymphocytes using a salting-out method. Samples were screened for the 31 most common CFTR gene mutations within the white popu- lation with the Cystic Fibrosis Diagnostic System kit (Abbott, Wiesbaden, Germany). ${ }^{13}$

\section{IVS8 poly(T)n, poly(TG)m, and M470V polymorphisms}

Characterization of the IVS8 poly(TG)m(T)n polymorphic sequence was carried out by PCR amplification of the junction between intron 8 and exon 9 (forward primer: 5'TGAAAATATCTGACAAACTC3'; reverse primer: 5'ATTTGGGTAGTGTGAAGGG3'), followed by DGGE analysis and direct DNA sequencing of the amplified DNA fragments using the Big Dye Terminator v1.1 Cycle Sequencing kit in an ABI PRISM 310 DNA automated sequencer with the appropriate sequencing analysis software (Applied Biosystems, Foster City, CA).12,13,27 M470V was studied by PCR amplification (forward primer: $5^{\prime}$ TCCTGAGCGTGATTTGATAA3'; reverse primer: 5'ATTTGGGTAGTGTGAAGGG3') (CFTR DGGE, Ingeny, The Netherlands), followed by direct DNA sequencing as above.

\section{Whole CFTR gene analysis}

The 27 CFTR exons and their boundaries were studied by DGGE and dHPLC, which currently achieves a 95\% mutation detection rate in European populations with CF. DNA samples with abnormal migration patterns were amplified by PCR with the same primers and sequenced for identification of the mutations as described above. ${ }^{13,27-29}$

\section{CFTR gene rearrangements}

CFTR gene rearrangements were screened by QFM-PCR. QFM-PCR is based on the simultaneous amplification of short CFTR gene fragments followed by comparison of the fluorescent profiles with control DNAs and quantification of the fluorescence of each amplicon. The amplification step of the PCR assay was stopped at the exponential phase, allowing the quantification of the number of alleles amplified. Three fluorescentlabeled multiplex PCR assays were developed to amplify all 27 CFTR exons, the promotor region, and the region containing the polyadenylation signal sequence. Primers were labeled with the fluorescent phosphoramidite 6-FAM dye. The detailed procedure is described elsewhere. ${ }^{22}$ Multiplex PCR-1 (MP1) amplified the promotor, the polyadenylation signal sequence, and exons 1-6a and 11. MP2 amplified 10 exons, 7-10 and 12-16. MP3 amplified 10 exons, 6b and 17a-24. Each MP assay contained control primer pairs (internal controls) that amplified short exonic fragments from other human genes located on different chromosomes, exon 4 of DSCR1 (chromosome 21 ) and exons 5, 7, or 8 of $F 9$ (X chromosome), depending on the MP used. Each experiment also included negative controls (samples with absence of deletions or duplications) and positive controls (samples with a known CFTR deletion). MP was performed in a $25-\mu \mathrm{L}$ reaction mixture using the Qiagen Multiplex PCR Kit (Qiagen, Courtaboeuf, France), with $300 \mathrm{ng}$ of genomic DNA and 0.1 to $0.8 \mu \mathrm{M}$ of primers. PCR cycling conditions were as follows: initial denaturation ( 15 minutes at $95^{\circ} \mathrm{C}$ ) followed by 21 cycles $\left(30\right.$ seconds at $95^{\circ} \mathrm{C}$; MP1/MP2: 30 seconds at $55^{\circ} \mathrm{C}$; or MP3: 30 seconds at $50^{\circ} \mathrm{C}$; extension: 45 seconds at $\left.72^{\circ} \mathrm{C}\right)$ and a final extension step (10 minutes at 
$72^{\circ} \mathrm{C}$ ). Primer concentrations and the number of amplification cycles were optimized to ensure that each PCR was still in the exponential phase of amplification. Purified PCR products (2 $\mu \mathrm{L}$ ) were added to a mixture containing $9.8 \mu \mathrm{L}$ of formamide and $0.2 \mu \mathrm{L}$ of GeneScan-500 Rox size standards (Applied Biosystems) and then separated on an ABI PRISM 310 DNA automated sequencer and analyzed using the GeneScan 3.1 software (Applied Biosystems) to obtain the electrophoregrams from each sample. Data were analyzed by superimposing fluorescent profiles of test and control DNAs followed by visual comparison of the peak heights of the corresponding amplicons, normalized against the F9 and/or DSCR1 peaks. The presence of a deletion was indicated by a decrease of approximately $50 \%$ in the height of the corresponding peak, whereas duplication showed an increase in signal intensity of approximately $50 \%$. To determine the copy number of each exon amplified, the peak height values from a sample were compared with one another and with those from controls, which gave a series of dosage quotients. Peak height data from samples and controls were imported into an Excel (Microsoft Corp, Redmond, WA) spreadsheet, and dosage quotients for pairs of exons in a sample were then calculated by dividing the ratio of the two exons' peak height from the sample by the corresponding ratio obtained from the controls.

\section{RESULTS}

\section{CFTR mutations and genotypes}

Genomic DNA from 45 patients with CAVD was extensively studied for CFTR mutations in the context of male infertility caused by CBAVD (42 patients) and CUAVD (3 patients). All patients had normal karyotypes and absence of Y-chromosome microdeletions, abnormalities of the urinary system, and clinical manifestations of CF. Analysis of the 31 most common CF mutations using a commercial kit (OLA, Abbott, Wiesbaden, Germany) identified seven different CFTR mutations in 32 patients $(71.1 \%)$. Study of the IVS8 poly(TG)m(T)n polymorphic loci identified at least one T5 allele in 25 patients (55.6\%). In total, the initial study allowed the identification of eight different mutations (seven CFTR mutations and the T5 allele) in 40 patients (88.9\%). Mutations were found in both alleles of 25 patients (55.6\%) (5 CFTR/CFTR, 17 CFTR/T5, 3 T5/T5) and in one allele of 15 patients (33.3\%) (10 CFTR/-, 5 $\mathrm{T} 5 /-$ ), whereas 5 patients (11.1\%) remained with no mutations identified (Tables 1 and 2).

In a second step, extensive analysis of the CFTR gene by DGGE and dHPLC was performed in DNA samples of those 40 patients with CAVD who after the initial screening had only one or no CFTR mutations detected. This revealed 17 further different CFTR mutations, thus increasing to 25 the number of different mutations detected. All 45 patients had at least one CFTR mutation or one T5 allele identified. Mutations were found in both alleles of 41 patients (91.1\%) (20 CFTR/CFTR, 19 CFTR/T5, 2 T5/T5) and in one allele of 4 patients (8.9\%) (3 CFTR/-, 1 T5/-) (Tables 1 and 2).

DNA samples of 12 patients with CAVD, still without a genotype that could explain the CAVD phenotype after DGGE/
dHPLC screening, were then selected to proceed for CFTR gene rearrangements analysis by QFM-PCR. These included two patients with one mild CFTR mutation, one patient with one T5 allele, seven patients whose CFTR mutations or T5 alleles could probably not explain the clinical phenotype (two cases with mild/mild CFTR mutations, three cases with one mild CFTR mutation and a T5 allele, and two cases with two T5 alleles), and two apparently homozygous patients for a mild CFTR mutation but in whom the homozygous status was not possible to confirm by familial studies (Table 2). Patients carrying one severe $\mathrm{CF}$ mutation in one allele were not selected for the CFTR rearrangements study, because it was not expected that another severe mutation would be detected (large deletion/insertion) in the absence of CF clinical manifestations. A large CFTR gene rearrangement CFTRdele2,3 $(21 \mathrm{~kb})$ was detected in a Ukrainian patient carrying the T5 allele. A duplex PCR assay was performed using specific primer pairs that annealed at both sides of the breakpoint junction (introns 1 and 3 of the CFTR gene) and at intron 3. A 243-base pair junction product was amplified, confirming the deletion of exons 2 and 3. This deletion spanned $21.08 \mathrm{~kb}$ of the CFTR gene and included approximately $25 \%$ of intron 1 , exon 2 , intron 2 , and exon 3 , and approximately $45 \%$ of intron 3 . CFTR gene rearrangements were thus found in 1 of 12 patients (8.3\%), which represents 1 of 45 of the total patients studied (2.2\%).

Overall, 26 different mutations were found in 45 patients with CAVD, 42 (93.3\%) with mutations in both alleles and 3 (6.7\%) with one mutation. Of the 26 different mutations found, 17 (65.4\%) were missense, 4 (15.4\%) were splice site, 2 (7.7\%) were nonsense, $2(7.7 \%)$ were deletions of a single amino acid, and $1(3.8 \%)$ was a large deletion (Table 1). Patients with two detected mutations were mostly compound heterozygotes $(38 / 42,90.5 \%)$, whereas four patients $(9.5 \%)$ were homozygotes for T5 allele (two cases), R334W, and S1235R (Table 2). The true homozygosity of the last two cases was confirmed by QFM-PCR. With the exception of one patient carrying two severe CF mutations (DeltaF508del/E831X), all other patients with two detected mutations (97.6\%) had a mild missense/splicing site mutation in one of the alleles. The most frequent genotype of compound heterozygotes was the association of a CF/CFTR mutation with the T5 allele, accounting for 20 of $38(52.6 \%)$ of the cases. Of them, deltaF508/T5 was the most frequent $(12 / 20,60 \%)$ genotype (Table 2$)$.

Three mutations were found as complex alleles (two or three sequence alterations associated in cis on the same allele), each in two patients, G576A-R668C, D443Y-G576A-R668C, and S1235R-T5 (one patient with T5 in homozygosity) (Table 2).

Three novel CFTR missense mutations were identified in three patients with CBAVD who were compound heterozygotes. P439S was identified in a 34 -year-old patient who carried a R334W mutation in the other chromosome. It results in a proline substitution by a serine at position 439 , located in the nucleotide-binding domain (NBD) 1 of the CFTR protein. V1108L was found in a 37-year-old patient who also carried the T5 allele. It causes the substitution of a valine by a leucine at position 1108 , located in the carboxyterminal transmembrane domain of the CFTR protein. 
Table 1

CFTR gene mutations and polymorphisms in patients with congenital absence of the vas deferens

\begin{tabular}{|c|c|c|c|c|c|}
\hline & Mutation & Location & Nucleotide alteration & Effect & Method \\
\hline 1 & CFTRdele2,3 & Exons 2-3 & Deletion of exons 2 and 3 & Frameshift & QFM-PCR \\
\hline 2 & $\mathrm{R} 117 \mathrm{H}$ & Exon 4 & $\mathrm{G} \rightarrow \mathrm{A}$ at 482 & AA substitution & 31 mutation panel \\
\hline 3 & P205S & Exon $6 a$ & $\mathrm{C} \rightarrow \mathrm{T}$ at 745 & AA substitution & DGGE/dHPLC \\
\hline 4 & L206W & Exon $6 a$ & $\mathrm{~T} \rightarrow \mathrm{G}$ at 749 & AA substitution & DGGE/dHPLC \\
\hline 5 & R258G & Exon $6 \mathrm{~b}$ & $\mathrm{~A} \rightarrow \mathrm{G}$ at 904 & AA substitution & DGGE/dHPLC \\
\hline 6 & $\mathrm{R} 334 \mathrm{~W}$ & Exon 7 & $\mathrm{C} \rightarrow \mathrm{T}$ at 1132 & AA substitution & 31 mutation panel \\
\hline 7 & T5 allele & Intron 8 & Deletion of $2 \mathrm{~T}$ at $1342-12$ to -6 & Aberrant splicing & DGGE/DNA sequencing \\
\hline 8 & P439S & Exon 9 & $\mathrm{C} \rightarrow \mathrm{T}$ at 1447 & AA substitution & DGGE/dHPLC \\
\hline 9 & $\mathrm{D} 443 \mathrm{Y}^{a}$ & Exon 9 & $\mathrm{G} \rightarrow \mathrm{T}$ at 1459 & AA substitution & DGGE/dHPLC \\
\hline 10 & I507del & Exon 10 & Deletion of $3 \mathrm{bp}$ at $1648-1653$ & AA deletion & 31 mutation panel \\
\hline 11 & DeltaF508 & Exon 10 & Deletion of $3 \mathrm{bp}$ at $1652-1655$ & AA deletion & 31 mutation panel \\
\hline 12 & G542X & Exon 11 & $\mathrm{G} \rightarrow \mathrm{T}$ at 1756 & Truncation & 31 mutation panel \\
\hline 13 & V562I & Exon 12 & $\mathrm{G} \rightarrow \mathrm{A}$ at 1816 & AA substitution & DGGE/dHPLC \\
\hline 14 & $\mathrm{G}^{2} 76 \mathrm{~A}^{a}$ & Exon 12 & $\mathrm{G} \rightarrow \mathrm{C}$ at 1859 & Aberrant splicing & DGGE/dHPLC \\
\hline 15 & D614G & Exon 13 & $\mathrm{~A} \rightarrow \mathrm{G}$ at 1973 & AA substitution & DGGE/dHPLC \\
\hline 16 & $\mathrm{R} 688 \mathrm{C}^{a}$ & Exon 13 & $\mathrm{C} \rightarrow \mathrm{T}$ at 2134 & AA substitution & DGGE/dHPLC \\
\hline 17 & V754M & Exon 13 & $\mathrm{G} \rightarrow \mathrm{A}$ at 2392 & AA substitution & DGGE/dHPLC \\
\hline 18 & E831X & Exon $14 \mathrm{a}$ & $\mathrm{G} \rightarrow \mathrm{T}$ at 2623 & Truncation & DGGE/dHPLC \\
\hline 19 & $3272-26 A>G$ & Intron $17 \mathrm{a}$ & $A \rightarrow G$ at $3272-26$ & Aberrant splicing & DGGE/dHPLC \\
\hline 20 & $2789+5 \mathrm{G} \rightarrow \mathrm{A}$ & Intron $14 \mathrm{~b}$ & $\mathrm{G} \rightarrow \mathrm{A}$ at $2789+5$ & Aberrant splicing & 31 mutation panel \\
\hline 21 & V1108L & Exon $17 \mathrm{~b}$ & $\mathrm{G} \rightarrow \mathrm{C}$ at 3454 & AA substitution & DGGE/dHPLC \\
\hline 22 & L1227S & Exon 19 & $\mathrm{~T} \rightarrow \mathrm{C}$ at 3812 & AA substitution & DGGE/dHPLC \\
\hline 23 & S1235R & Exon 19 & $\mathrm{~T} \rightarrow \mathrm{G}$ at 3837 & AA substitution & DGGE/dHPLC \\
\hline 24 & P1290S & Exon 20 & $\mathrm{C} \rightarrow \mathrm{T}$ at 4000 & AA substitution & DGGE/dHPLC \\
\hline 25 & N1303K & Exon 21 & $\mathrm{C} \rightarrow \mathrm{G}$ at 4041 & AA substitution & 31 mutation panel \\
\hline \multirow[t]{2}{*}{26} & E1401K & Exon 23 & $\mathrm{G} \rightarrow \mathrm{A}$ at 4333 & AA substitution & DGGE/dHPLC \\
\hline & Polymorphisms & & & & \\
\hline 1 & TG repeats & Intron 8 & $9-13$ copies at $1342-12$ to -35 & Sequence variation & DGGE/DNA sequencing \\
\hline 2 & M470V & Exon 10 & A or $\mathrm{G}$ at 1540 & Sequence variation & DNA sequencing \\
\hline 3 & $125 \mathrm{G} / \mathrm{C}$ & Exon 1 & $\mathrm{G} \rightarrow \mathrm{C}$ at 125 & Sequence variation & DGGE/dHPLC \\
\hline 4 & $1001+11 \mathrm{~T} / \mathrm{C}$ & Intron $6 \mathrm{~b}$ & $\mathrm{C} \rightarrow 4 \mathrm{~T}$ at $1001+11$ & Sequence variation & DGGE/dHPLC \\
\hline 5 & 1716G/A & Exon 10 & $\mathrm{G} \rightarrow \mathrm{A}$ at 1716 & Sequence variation & DGGE/dHPLC \\
\hline 6 & $1899-136 \mathrm{~T} / \mathrm{G}$ & Intron 12 & $\mathrm{~T} \rightarrow \mathrm{G}$ at $1899-136$ & Sequence variation & DGGE/dHPLC \\
\hline 7 & $\mathrm{~T} 854 \mathrm{~T}$ & Exon $14 \mathrm{a}$ & $\mathrm{T} \rightarrow \mathrm{G}$ at 2694 & Sequence variation & DGGE/dHPLC \\
\hline 8 & $3601-65 \mathrm{C} / \mathrm{A}$ & Intron 18 & $C \rightarrow A$ at $3601-65$ & Sequence variation & DGGE/dHPLC \\
\hline 9 & $4521 \mathrm{G} / \mathrm{A}$ & Exon 24 & $\mathrm{G} \rightarrow \mathrm{A}$ at 4521 & Sequence variation & DGGE/dHPLC \\
\hline
\end{tabular}

QFM-PCR, semiquantitative fluorescent multiplex polymerase chain reaction; bp, base pair; DGGE, denaturing gradient gel electrophoresis; dHPLC, denaturing high-performance liquid chromatography.

${ }^{a}$ Missense mutations linked on the same allele, G576A-R688C and D443Y-G576A-R688C, were present in two patients with CBAVD each.

CFTR mutations: severe (bold); novel (italics).

E1401K was found in a 37-year-old patient who carried a deltaF508 mutation in the other chromosome. It leads to the substitution of a glutamic acid by a lysine in (NBD) 2 at its carboxyterminal side (Tables 1 and 2). None of these mutations were detected in 200 chromosomes from 100 healthy individuals and in 10 chromosomes from 5 patients with CF.
T5 was the most frequent mutation found, accounting for 28 of $90(31.1 \%)$ of the alleles (Table 3 ). (TG) $12 \mathrm{~T} 5$ was the most frequent associated haplotype $(18 / 28,64.4 \%)$, followed by the (TG) $11 \mathrm{~T} 5(5 / 28,17.8 \%)$ and (TG)13T5 (5/28, 17.8\%) alleles (Table 2). DeltaF508 was the second most common mutation, representing 21 (23.3\%) of total alleles, followed by R334W (6, 
Table 2

CFTR genotypes identified in patients with congenital absence of the vas deferens

\begin{tabular}{|c|c|c|c|c|c|c|c|}
\hline & & & & & & & \\
\hline CFTR $\mathrm{m}$ & enotypes & {$[(\mathrm{TG})$} & otype & & & $\mathrm{N}$ & $\%$ \\
\hline$\underline{\text { DeltaF508 }}$ & & (TG)10T9 & (TG)12T5 & M & V & 11 & 24.4 \\
\hline$\underline{\text { DeltaF508 }}$ & & (TG) $10 \mathrm{~T} 9$ & (TG)11T5 & M & M & 1 & 2.2 \\
\hline$\underline{\text { DeltaF508 }}$ & $\underline{\mathrm{R} 117 \mathrm{H}}$ & (TG)10T9 & (TG) $10 \mathrm{~T} 7$ & M & M & 2 & 4.4 \\
\hline$\underline{\text { G542X }}$ & & (TG) $10 \mathrm{~T} 9$ & (TG)12T5 & M & $\mathrm{V}$ & $2^{a}$ & 4.4 \\
\hline$\underline{\text { DeltaF508 }}$ & $\underline{\mathrm{R} 334 \mathrm{~W}}$ & (TG) $10 \mathrm{~T} 9$ & (TG) $11 \mathrm{~T} 7$ & M & $\mathrm{V}$ & 1 & 2.2 \\
\hline$\underline{\text { DeltaF508 }}$ & D443Y-G576A-R668C & (TG) $10 \mathrm{~T} 9$ & (TG) $10 \mathrm{~T} 7$ & M & M & 1 & 2.2 \\
\hline$\underline{\text { DeltaF508 }}$ & D614G & (TG) $10 \mathrm{~T} 9$ & (TG) $11 \mathrm{~T} 7$ & M & $\mathrm{V}$ & 1 & 2.2 \\
\hline DeltaF508 & E831X & (TG) $10 \mathrm{~T} 9$ & (TG) $11 \mathrm{~T} 7$ & M & $\mathrm{V}$ & 1 & 2.2 \\
\hline$\underline{\text { DeltaF508 }}$ & L1227S & (TG) $10 \mathrm{~T} 9$ & (TG) $11 \mathrm{~T} 7$ & M & $\mathrm{M}$ & 1 & 2.2 \\
\hline$\underline{\text { DeltaF508 }}$ & E1401K & (TG) $10 \mathrm{~T} 9$ & (TG) $11 \mathrm{~T} 7$ & M & $\mathrm{V}$ & 1 & 2.2 \\
\hline$\underline{\text { I507del }}$ & D614G & (TG) $11 \mathrm{~T} 7$ & (TG)10T7 & M & $\mathrm{V}$ & 1 & 2.2 \\
\hline$\underline{\mathrm{N} 1303 \mathrm{~K}}$ & L206W & (TG) $10 \mathrm{~T} 9$ & (TG)9T9 & M & $\mathrm{M}$ & 1 & 2.2 \\
\hline $\mathrm{R} 117 \mathrm{H}$ & $\underline{\text { P205S }}$ & (TG) $11 \mathrm{~T} 7$ & (TG) $10 \mathrm{~T} 7$ & M & $\mathrm{V}$ & 1 & 2.2 \\
\hline$\underline{\mathrm{R} 117 \mathrm{H}}$ & $\underline{\mathrm{R} 334 \mathrm{~W}}$ & (TG) $10 \mathrm{~T} 7$ & (TG) $11 \mathrm{~T} 7$ & M & $\mathrm{V}$ & 1 & 2.2 \\
\hline$\underline{\mathrm{R} 334 \mathrm{~W}}$ & P439S & (TG)11T7 & (TG) $11 \mathrm{~T} 7$ & M & $\mathrm{V}$ & 1 & 2.2 \\
\hline$\underline{\mathrm{R} 334 \mathrm{~W}}$ & $\underline{\mathrm{R} 334 \mathrm{~W}^{b}}$ & (TG) $11 \mathrm{~T} 7$ & (TG) $11 \mathrm{~T} 7$ & $\mathbf{V}$ & $\mathrm{V}$ & 1 & 2.2 \\
\hline $\mathrm{R} 334 \mathrm{~W}$ & V562I & (TG) $11 \mathrm{~T} 7$ & (TG)11T5 & $\mathbf{V}$ & M & 1 & 2.2 \\
\hline D443Y-G576A-R668C & $3272-26 \mathrm{~A} \rightarrow \mathrm{G}$ & (TG) $10 \mathrm{~T} 7$ & (TG) $10 \mathrm{~T} 7$ & M & M & 1 & 2.2 \\
\hline G576A-R668C & V754M $\mathrm{M}^{b}$ & (TG) $10 \mathrm{~T} 7$ & (TG) $11 \mathrm{~T} 7$ & M & M & 1 & 2.2 \\
\hline S1235R & $\mathrm{S} 1235 \mathrm{R}^{b}$ & (TG)13T5 & (TG)13T5 & M & M & 1 & 2.2 \\
\hline $2789+5 \mathrm{G} \rightarrow \mathrm{A}$ & $\mathrm{S} 1235 \mathrm{R}^{b}$ & (TG) $10 \mathrm{~T} 7$ & (TG)13T5 & M & M & 1 & 2.2 \\
\hline $3272-26 \mathrm{~A} \rightarrow \mathrm{G}$ & P1290S & (TG) $11 \mathrm{~T} 7$ & (TG) $10 \mathrm{~T} 7$ & M & V & 1 & 2.2 \\
\hline P205S & & (TG) $11 \mathrm{~T} 7$ & (TG)12T5 & V & $\mathrm{V}$ & 1 & 2.2 \\
\hline G576A-R668C & $b$ & (TG) $10 \mathrm{~T} 7$ & (TG)11T5 & M & M & 1 & 2.2 \\
\hline V1108L & $b$ & (TG) $11 \mathrm{~T} 7$ & (TG)11T5 & $\mathrm{V}$ & $\mathrm{M}$ & 1 & 2.2 \\
\hline N1303K & & (TG)10T9 & (TG)12T5 & M & V & 1 & 2.2 \\
\hline $3272-26 A \rightarrow G$ & $b$ & (TG) $10 \mathrm{~T} 7$ & (TG) 12T5 & M & $\mathrm{V}$ & 1 & 2.2 \\
\hline CFTRdele2,3 & $b$ & (TG)11T7 & (TG)13T5 & $\mathrm{V}$ & M & 1 & 2.2 \\
\hline & $b$ & (TG)11T5 & (TG) 12T5 & M & $\mathrm{V}$ & 1 & 2.2 \\
\hline & $b$ & (TG)13T5 & (TG) 12T5 & M & $\mathrm{V}$ & 1 & 2.2 \\
\hline DeltaF508 & - & (TG) $10 \mathrm{~T} 9$ & (TG) $11 \mathrm{~T} 7$ & M & $\mathrm{V}$ & $1^{a}$ & 2.2 \\
\hline L206W & $-^{b}$ & (TG)9T9 & (TG) $11 \mathrm{~T} 7$ & M & $\mathrm{V}$ & 1 & 2.2 \\
\hline R258G & $-^{b}$ & (TG)11T7 & (TG) $11 \mathrm{~T} 7$ & $\mathrm{~V}$ & $\mathrm{~V}$ & 1 & 2.2 \\
\hline
\end{tabular}

${ }^{a}$ CUAVD.

${ }^{b}$ Subjected to QFM-PCR analysis.

CFTR mutations: detected by panel CF screening (underlined); severe (bold); novel (italics).

$6.7 \%)$. These three mutations led to the identification of 55 of $90(61.1 \%)$ of the total alleles (Tables 2 and 3). The allelic frequency of the other mutations was $4.4 \%$ for R117H, G576A, and R668C, 3.3\% for S1235R and 3272-26A $\rightarrow \mathrm{G}$, and $2.2 \%$ for P205S, L206W, D443Y, G542X, D614G, and N1301K, whereas the remaining 12 mutations were present in single patients (Table 3).

\section{CFTR polymorphisms}

The extensive CFTR gene screening performed in 40 DNA samples by DGGE/dHPLC revealed the presence of seven polymor- phisms, of which one is novel. The allelic frequencies of these sequence variations were $21.3 \%$ (17/80) for 2694T/G, 20\% (16/80) for $1001+11 \mathrm{~T} / \mathrm{C}, 18.8 \%$ (15/80) for $4521 \mathrm{G} / \mathrm{A}, 6.3 \%(5 / 80)$ for $1899-136 \mathrm{~T} / \mathrm{G}$, and $1.3 \%$ for $125 \mathrm{G} / \mathrm{C}, 1716 \mathrm{G} / \mathrm{A}$, and $3601-65 \mathrm{C} / \mathrm{A}^{1}$ (Table 1). The novel polymorphism 1899-136T/C was detected by DGGE analysis in five heterozygous patients with CBAVD. Analysis of 114 alleles from 57 healthy individuals showed that the $\mathrm{C}$ variant was present in $30(26.3 \%)$ of the total alleles studied (6 cases in homozygotes and 18 cases in heterozygotes).

The missense M470V polymorphism was evaluated in all $45 \mathrm{pa}-$ tients with CAVD (Table2). The allelic frequency of the M470 variant 
Table 3

Allelic frequencies of CFTR mutations in patients with congenital absence of the vas deferens

\begin{tabular}{|c|c|c|c|c|c|c|c|c|}
\hline & \multirow[b]{4}{*}{ Mutations } & \multicolumn{3}{|c|}{ CBAVD } & \multicolumn{2}{|c|}{ CUAVD } & \multicolumn{2}{|c|}{ Total } \\
\hline & & \multirow{3}{*}{$\begin{array}{l}\text { Patients } \\
\text { Alleles }\end{array}$} & \multicolumn{2}{|c|}{42} & \multicolumn{2}{|c|}{3} & \multicolumn{2}{|c|}{45} \\
\hline & & & \multicolumn{2}{|c|}{84} & \multicolumn{2}{|c|}{6} & \multicolumn{2}{|c|}{90} \\
\hline & & & $\mathrm{N}$ & $\%$ & $\mathrm{~N}$ & $\%$ & $\mathrm{~N}$ & $\%$ \\
\hline 1 & T5 allele & & $26^{a}$ & 31 & 2 & 33.3 & 28 & 31.1 \\
\hline 2 & DeltaF508 & & 20 & 23.8 & 1 & 16.7 & 21 & 23.3 \\
\hline 3 & R334W & & $6^{a}$ & 7.1 & 0 & 0 & 6 & 6.7 \\
\hline 4 & $\mathrm{R} 117 \mathrm{H}$ & & 4 & 4.8 & 0 & 0 & 4 & 4.4 \\
\hline 5 & G576A & & $4^{b}$ & 4.8 & 0 & 0 & 4 & 4.4 \\
\hline 6 & $\mathrm{R} 688 \mathrm{C}$ & & $4^{b}$ & 4.8 & 0 & 0 & 4 & 4.4 \\
\hline 7 & S1235R & & $3^{a}$ & 3.6 & 0 & 0 & 3 & 3.3 \\
\hline 8 & $3272-26 \mathrm{~A} \rightarrow \mathrm{G}$ & & 3 & 3.6 & 0 & 0 & 3 & 3.3 \\
\hline 9 & P205S & & 2 & 2.4 & 0 & 0 & 2 & 2.2 \\
\hline 10 & L206W & & 2 & 2.4 & 0 & 0 & 2 & 2.2 \\
\hline 11 & D443Y & & $2^{b}$ & 2.4 & 0 & 0 & 2 & 2.2 \\
\hline 13 & D614G & & 2 & 2.4 & 0 & 0 & 2 & 2.2 \\
\hline 14 & N1303K & & 2 & 2.4 & 0 & 0 & 2 & 2.2 \\
\hline 12 & G542X & & 0 & 0 & 2 & 33.3 & 2 & 2.2 \\
\hline 15 & R258G & & 1 & 1.2 & 0 & 0 & 1 & 1.1 \\
\hline 16 & P439S & & 1 & 1.2 & 0 & 0 & 1 & 1.1 \\
\hline 17 & I507del & & 1 & 1.2 & 0 & 0 & 1 & 1.1 \\
\hline 18 & V562I & & 1 & 1.2 & 0 & 0 & 1 & 1.1 \\
\hline 19 & V754M & & 1 & 1.2 & 0 & 0 & 1 & 1.1 \\
\hline 20 & E831X & & 1 & 1.2 & 0 & 0 & 1 & 1.1 \\
\hline 21 & $2789+5 \mathrm{G} \rightarrow \mathrm{A}$ & & 1 & 1.2 & 0 & 0 & 1 & 1.1 \\
\hline 22 & V1108L & & 1 & 1.2 & 0 & 0 & 1 & 1.1 \\
\hline 23 & L1227S & & 1 & 1.2 & 0 & 0 & 1 & 1.1 \\
\hline 24 & P1290S & & 1 & 1.2 & 0 & 0 & 1 & 1.1 \\
\hline 25 & E1401K & & 1 & 1.2 & 0 & 0 & 1 & 1.1 \\
\hline 26 & CFTRdele2,3 & & 1 & 1.2 & 0 & 0 & 1 & 1.1 \\
\hline
\end{tabular}

CBAVD, congenital bilateral absence of the vas deferens; CUAVD, congenital unilateral absence of the vas deferens.

${ }^{a}$ Includes homozygotes. ${ }^{b}$ Missense mutations linked on the same allele, G576A-R688C and D443Y-G576A-R688C, were present in two patients with CBAVD each.

(A at nucleotide 1540 ) was $58.9 \%(53 / 90)$ and of the $V 470$ variant ( $G$ at nucleotide 1540) was $41.1 \%$ (37/90). Most of the patients with the V470 variant had an associated T5 allele $(21 / 37,56.8 \%)$, especially in combination with the (TG) $12 \mathrm{~T} 5$ haplotype (17/21, 81\%).

\section{DISCUSSION}

The strategy for mutation detection in the 45 patients with CAVD included an initial screening for the 31 most frequent CFTR mutations found in patients with CF and the analysis of the IVS8 poly(T)n alleles with two associated polymorphisms: poly(TG)m and M470V. This allowed the detection of 8 differ- ent mutations in 40 patients (88.9\%), with $25(55.6 \%)$ showing mutations in both alleles (5 CFTR/CFTR, 11.1\%). In a second step, DNA samples from 40 patients with only one or no CFTR mutations were subjected to a complete screening of the 27 exons and their flanking intronic regions by DGGE and dHPLC. This identified 17 further different mutations, thus increasing to 25 the number of different mutations and to 45 the number of patients who had at least one mutation, with 41 (91.1\%) showing mutations in both alleles (20 CFTR/CFTR, $44.4 \%)$. Twelve patients who remained without a genotype justificative for CAVD were then selected to proceed for the study of CFTR gene rearrangements by QFM-PCR. Large 
CFTR gene rearrangements are not detectable using conventional PCR-based screening techniques, because the amplification of a nondeleted allele masks the lack of a PCR product corresponding to the deletion site..$^{21,22,24}$ This detected a CFTRdele2,3 $(21 \mathrm{~kb})$ deletion in a Ukrainian emigrant with CBAVD and heterozygous for the T5 allele. This large deletion was previously described in $5 \%$ of Slavic patients with CF from Central and Eastern Europe and was predicted to cause a truncated CFTR protein lacking all functional domains. ${ }^{30,31}$ In the present case, the combination of CFTRdele2,3 $(21 \mathrm{~kb})$ with the T5 allele in trans is thus expected to result in reduced CFTR channel function. Large CFTR gene rearrangements were previously described to account for $1.3 \%$ of all $\mathrm{CF}$ mutations and for $16 \%$ to $20 \%$ of unidentified CF mutations, having also been found in approximately $2 \%$ of patients with CBAVD. ${ }^{21-25}$ Our present results are in accordance with those findings, because CFTR gene rearrangements were found in 1 of 45 patients (2.2\%) with CAVD. QFM-PCR also confirmed the true CFTR homozygosity of two CAVD cases (R334W and S1235R), because the determination of CFTR copy numbers by semiquantitative PCR also allows the discrimination between true homozygotes and compound heterozygotes for a CFTR mutation.

In total, 26 different mutations were found, with at least one mutation being detected in all 45 patients with CAVD, 42 (93.3\%) showing mutations in both alleles (20 CFTR/CFTR, 44.4\%). In comparison with previous studies, this is the highest mutation frequency reported for patients with CAVD ${ }^{5,6,13,30,32-37}$ (Table 4). The three patients with CAVD (6.7\%) who remained with only one mutation detected might be explained by mutations located outside of the analyzed regions, such as sequence alterations in intronic regulatory regions or mutations in other disease-causing genes. This could result in a CFTR protein with normal structure but with low levels of expression, thus chang-

Table 4

Comparative analysis of the frequency of patients with congenital absence of the vas deferens with at least one CFTR mutation or T5 allele after complete screening of the CFTR gene

\begin{tabular}{lrrrr}
\hline & \multicolumn{3}{c}{ Patients } \\
\cline { 2 - 4 } & \multicolumn{3}{c}{$\begin{array}{c}\text { At least } \\
\text { one mutation }\end{array}$} \\
\cline { 3 - 4 } Countries & $\mathrm{N}$ & $\mathrm{N}$ & $\%$ & References \\
\hline Iran & 113 & 57 & 50 & 37 \\
Brasil & 17 & 10 & 59 & 34 \\
Canada & 74 & 49 & 66 & 5 \\
Greece & 14 & 10 & 71 & 32 \\
Spain & 134 & 103 & 77 & 33 \\
Turkey & 51 & 40 & 78 & 35 \\
Germany & 106 & 89 & 84 & 30 \\
France & 327 & 284 & 87 & 6 \\
Portugal & 45 & 45 & 100 & 13,36, PS \\
\hline PS, present & & &
\end{tabular}

PS, present study. ing the development of susceptible tissues such as the vas deferens and epididymis. ${ }^{9,38}$

As expected for an incomplete CF phenotype, the majority of the 26 different mutations detected corresponded to amino acid changes (69.2\%) and splice site mutations (11.5\%), which cause a mild CFTR dysfunction. Despite the high heterogeneity of the mutations found in Portuguese patients with CAVD, three were the most frequent mutations (T5, deltaF508, and $\mathrm{R} 334 \mathrm{~W})$, being present in 37 of 45 cases (82\%) with 61\% (55/ 90) allelic frequency. The T5 allele was the most frequent mutation, with $31 \%$ allelic frequency, which is one of the higher frequencies in comparison with previous studies. In Portugal, the T5 allele was found with an allelic frequency of $3 \%$ in fertile males and of $5 \%$ in the general population. ${ }^{13}$ As in other studies, deltaF508 was the second most frequent mutation, with an allelic frequency of $23 \%$. However, and contrary to all other studies, the third most frequent mutation was R334W, with $7 \%$ allelic frequency ${ }^{5,6,13,30,32-44}$ (Table 5).

Compound heterozygosity was found in $91 \%$ (38/42) of the cases with two mutations. The most frequent compound genotype was a combination between a CF/CFTR mutation and the T5 allele (20/38, 53\%), with deltaF508/T5 being responsible for $60 \%(12 / 20)$ of these cases. The high frequency of mild or very mild CFTR mutations in patients with CAVD adds further evidence that the vas deferens is one of the tissues most susceptible to the effect of changes in CFTR activity. In fact, previous studies have shown that healthy individuals produce less functional CFTR protein in the reproductive tract than in the respiratory epithelium, because the splicing efficiency of exon 9 is lower regardless of the IVS8 poly(T)n genotype. In the presence of mild CFTR mutations, there might be enough CFTR protein to prevent disease in other organs, but the additive effect of the T5 allele specifically affects the reproductive tract and results in the production of a nonfunctional protein, thus explaining why CAVD does not necessarily coincide with pulmonary or pancreatic manifestations of CF.9,45,46

Although a particular sequence variation may not have deleterious consequences when present in isolation, the combination of such variations in cis might result in a less functional or even insufficient CFTR protein. Studies have shown that intronic IVS8 poly(TG)m and exonic (M470V) cis-acting elements affect the splicing efficiency of the CFTR gene bearing the $\mathrm{T} 5$ allele. The (TG) $\mathrm{m}$ tract variant located in intron 8 has a predominant role in the exclusion of exon 9 from CFTR transcripts, because higher numbers of TG repeats (12/13) increase the penetrance of the T5 allele. ${ }^{14,17-19}$ In accordance with these findings, the present study also showed that the T5 allele was mainly combined with the TG12 and TG13 variants (82\%). The M470V polymorphism located in exon 10 has also been reported as a modifier element of the penetrance of the T5 allele, with the V470 variant being involved in lower normal CFTR protein levels. ${ }^{14,15,19}$ In the present study, the V470 variant was found in 34 of 45 patients (76\%), with an allelic frequency of $41 \%$ (37/90). Although the phase of these alleles was not established, most patients with CAVD with T5 allele were associated with the V470 variant (18/28, 64.3\%). As expected 
Table 5

Comparative analysis of CFTR mutation allelic frequencies (\%) in patients with congenital absence of the vas deferens

\begin{tabular}{|c|c|c|c|c|c|c|}
\hline Countries & Patients & T5 allele & DeltaF508 & R334W & $\mathrm{R} 117 \mathrm{H}$ & References \\
\hline Argentina & 36 & NA & 20.8 & NA & 5.6 & 43 \\
\hline Austria & 22 & NA & 13.6 & NA & 9.1 & 44 \\
\hline Italy & 12 & 8.3 & 29.2 & NA & 4.2 & 39 \\
\hline The Netherlands & 21 & 9.5 & 19.0 & NA & 21.4 & 38 \\
\hline Germany & 106 & 12.3 & 26.4 & 0.5 & 11.3 & 30 \\
\hline Greece & 14 & 14.3 & 14.3 & NA & NA & 32 \\
\hline France & 800 & 16.3 & 21.8 & NA & 4.4 & 6 \\
\hline United States & 92 & 17.9 & 21.2 & NA & 2.2 & 41 \\
\hline Canada & 74 & 18.2 & 16.9 & 1.4 & 6.1 & 5 \\
\hline Turkey & 51 & 19.6 & 2.9 & NA & NA & 35 \\
\hline Brazil & 17 & 20.6 & 11.7 & NA & 2.9 & 34 \\
\hline Spain & 134 & 20.9 & 16.0 & 0.4 & 3.0 & 33 \\
\hline Iran & 113 & 25.7 & 12.4 & 0.9 & 3.5 & 37 \\
\hline Egypt & 16 & 43.7 & 6.2 & NA & NA & 40 \\
\hline Taiwan & 27 & 44.4 & NA & NA & NA & 42 \\
\hline Portugal & 45 & 31.1 & 23.3 & 6.7 & 4.4 & 13,36, PS \\
\hline
\end{tabular}

NA, not available; PS, present study.

by previous reports, a strong association was observed among the (TG) 12 (T) 5 , V470 allele, and the CAVD phenotype. ${ }^{14,19}$ Homozygosity for the T5 allele is a less frequent genotype, causing exon 9 skipping in approximately $90 \%$ of the transcripts. ${ }^{9,10}$ In the present study, two patients were homozygous for the T5 allele in association with TG12/V470 variants. This suggests that an undetected mutation might be located in cis in one allele or the association of other genetic factor could be responsible for the CBAVD phenotype.

Patients with CAVD carry CFTR genotypes composed of a severe and a mild mutation or two mild mutations. ${ }^{2,9}$ However, one Portuguese patient with CBAVD, without clinical manifestation of CF, was found to be a compound heterozygote for two severe CFTR mutations, deltaF508/E831X. This genotype was previously associated with a CF phenotype, with pancreatic insufficiency. ${ }^{8}$ A Turkish patient with CBAVD was also reported to be a compound heterozygote for the same E831X mutation and the truncating mutation 1677delTA. ${ }^{35} \mathrm{It}$ is possible that the association of the E831X mutation with a CF mutation confers an unusually mild phenotype, because the disease phenotype may be ameliorated through modulation of the CFTR genotype by external/internal factors or modifier genes. ${ }^{47}$ In addition, E831X mutation is located at the first nucleotide of exon 14a and may alter exon 14a splicing. The exon 14a skipping may generate a mutant protein that keeps residual function. Therefore, E831X may not be considered as a classic severe mutation.

Three novel missense mutations (E1401K, P439S, and V1108L), not detected in the general population or in patients with $\mathrm{CF}$, were here first described in patients with CAVD. Al- though the pathogenicity of these mutations is still being assessed by expression and functional in vitro studies, the combination of the amino acid substitutions with other mutations (deltaF508/E1401K, R334W/P439S, V1108L/T5) might be responsible for the CBAVD phenotype. In fact, they occur in highly conserved regions of the CFTR protein, which share $100 \%$ amino acid sequence homology between species ${ }^{48}$ and affect the NBD1, NBD2, and transmembrane regions of the protein, which are known to regulate chloride conductance and permeability. ${ }^{49-51} \mathrm{P} 439 \mathrm{~S}$ was previously reported in a child with $\mathrm{CF}$ with pancreatic insufficiency and mild lung disease, in association with the P439S/R688C genotype. ${ }^{52}$ The E1401K mutation occurs at a position in which other mutations, E1401X and E1401A, have been described in patients with CF with pancreatic insufficiency. ${ }^{8}$

Some difficulties in defining CF or CAVD-causing mutations were observed with some missense mutations.6,27 G576A and R668C have been found independently, in pairs, or combined with the D443Y mutation on the same chromosome in patients with a CF-related syndrome. In accordance with previous studies, we expected that G576A and R668C were located in cis in two patients and combined with D443Y in the same chromosome in two patients.69,12 Although initially described as polymorphisms, ${ }^{27}$ they were later considered mild mutations associated with the CBAVD phenotype when combined in trans with the severe deltaF508 mutation. ${ }^{33}$ However, our present results suggest they might also cause the CAVD phenotype when associated with other mild CFTR mutations, because three of four patients carrying these complex alleles harbored a mild or very mild mutation in the other chromosome (D443Y-G576A-R668C/3272-26A $\rightarrow \mathrm{G}$, 
G576A-R668C/V754M, G576A-R668C/T5), and only one case was associated with a severe mutation (DeltaF508del/D443YG576A-R668C).

In regard to the S1235R mutation, in this study it was found in homozygosity in one patient $(2.2 \%)$ and in compound heterozygosity with a mild CFTR mutation $(2789+5 \mathrm{G} \rightarrow \mathrm{A} / \mathrm{S} 1235 \mathrm{R})$ in one patient (2.2\%). In both cases, S1235R was in cis with the haplotype (TG)13T5. Homozygosity for the S1235R mutation is first described here, whereas compound heterozygosity between S1235R and another CFTR mutation (DeltaF508del) was previously described with a frequency of $0.08 \%$ in patients with variable CF phenotypic manifestations ${ }^{6}$ or of $0.12 \%$ to $0.98 \%$ in patients with CAVD. ${ }^{2,6}$ Although there is some controversy whether T5 homozygosity explains the CAVD phenotype, the T5/T5 genotype was found with a frequency of $6.7 \%$ (Table 2), similar to that previously reported $(1.1 \%-10.4 \%) .2,5,30,33,35,37,41$ Because the penetrance of the $\mathrm{T} 5$ allele might be increased when there is an association between the T5 allele and the (TG) 13 tract, the combination of S1235R with (TG)13T5 in homozygosity or in compound heterozygosity with another CFTR mutation might be responsible for the CBAVD phenotype, although the primary pathogenic factor, the $\mathrm{T} 5$ allele or the S1235R mutation, remains to be evaluated.

In conclusion, when patients display one or no mutations in the initial CFTR panel screening, all 27 exons should be analyzed (DGGE, single-strand conformation polymorphism, dHPLC, and DNA sequencing). If still negative, the study of large CFTR gene rearrangements should then be considered. In CF mutation/T5 or CFTR mutation/T5 compound heterozygotes, analysis of the haplotypes associated with the T5 allele is of interest because (TG)m repeats and M470V variants help in predicting the pathogenesis of the T5 allele. In patients with CAVD with one or two mutations identified, who undergo the assisted reproduction technique, the female partners should also be screened for mutations in the CFTR gene. If the female partner has at least one mutation, genetic counseling should be offered and patients informed about the possibility of prenatal or preimplantation genetics diagnosis.

\section{ACKNOWLEDGMENTS}

This work was partially supported by the Foundation for Science and Technology of the Ministry for Science, Technology and Superior Education (POCI/SAU-MMO/60709/04, $60555 / 04,59997 / 04$; UMIB). The sponsors of the study had no role in the study design, data collection, data analysis, data interpretation, or writing of the report.

\section{References}

1. Anguiano A, Oates RD, Amos JA, Dean M, et al. Congenital bilateral absence of the vas deferens. A primarily genital form of cystic fibrosis. J Am Med Assoc 1992;267: 1794-1797.

2. Chillón M, Casals T, Mercier B, Bassas L, et al. Mutations in the cystic fibrosis gene in patients with congenital absence of the vas deferens. N Engl J Med 1995;332:14751480 .

3. Mickle J, Milunsky A, Amos JA, Oates RD. Congenital unilateral absence of the vas deferens: a heterogeneous disorder with two distinct subpopulations based upon aetiology and mutational status of the cystic fibrosis gene. Hum Reprod 1995;10: 1728-1735.
4. Meschede D, Dworniczak B, Behre HM, Kliesch S, et al. CFTR gene mutations in men with bilateral ejaculatory-duct obstruction and anomalies of the seminal vesicles. Am J Hum Genet 1997;61:1200-1202.

5. Mak V, Zielenski J, Tsui L-C, Durie P, et al. Proportion of cystic fibrosis gene mutations not detected by routine testing in men with obstructive azoospermia. J Am Med Assoc 1999;281:2217-2224.

6. Claustres M, Guittard C, Bozon D, Chevallier F, et al. Spectrum of CFTR mutations in cystic fibrosis and in congenital absence of the vas deferens in France. Hum Mutat 2000;216:143-156.

7. McCallum TJ, Milunsky JM, Munarriz R, Carson R, et al. Unilateral renal agenesis associated with congenital bilateral absence of the vas deferens: phenotypic findings and genetic considerations. Hum Reprod 2001;16:282-288.

8. Cystic fibrosis mutation database, 2006. Available at: http://www.genet.sickkids. on.ca/cftr/. Accessed September 4, 2006.

9. Claustres M. Molecular pathology of the CFTR locus in male infertility. Reprod Biomed Online 2004;10:14-41.

10. Chu C-S, Trapnell BC, Curristin SM, Cutting GR, et al. Genetic basis of variable exon 9 skipping in cystic fibrosis transmembrane conductance regulator gene mRNA. Nat Genet 1993;3:151-156.

11. Kiesewetter S, Macek M, Davis C, Curristin SM, et al. A mutation in CFTR produces different phenotypes depending on chromosomal background. Nat Genet 1993;5: 274-278.

12. Costes B, Girodon E, Ghanem N, Flori E, et al. Frequent occurrence of the CFTR intron 8 (TG)n 5T allele in men with congenital bilateral absence of the vas deferens. Eur J Hum Genet 1995;3:285-293.

13. Grangeia A, Niel F, Carvalho F, Fernandes S, et al. Characterization of cystic fibrosis conductance transmembrane regulator gene mutations and IVS8 poly-T variants in Portuguese patients with congenital absence of the vas deferens. Hum Reprod 2004; 19:2502-2508.

14. Cuppens H, Lin W, Jaspers M, Costes B, et al. Polyvariant mutant cystic fibrosis transmembrane conductance regulator genes. The polymorphic $(\mathrm{Tg}) \mathrm{m}$ locus explains the partial penetrance of the T5 polymorphism as a disease mutation. J Clin Invest 1998;101:487-496.

15. de Meeus A, Guittard C, Desgeorges M, Carles S, et al. Linkage disequilibrium between the M470V variant and the IVS8 polyT alleles of the CFTR gene in CBAVD. J Med Genet 1998;35:594-596.

16. Niksic M, Romano M, Buratti E, Pagani F, et al. Functional analysis of cis-acting elements regulating the alternative splicing of human CFTR exon 9. Hum Mol Genet 1999;8:2339-2349.

17. Pagani F, Buratti E, Stuani C, Romano M, et al. Splicing factors induce cystic fibrosis transmembrane regulator exon 9 skipping through a nonevolutionary conserved intronic element. J Biol Chem 2000;275:21041-21047.

18. Hefferon TW, Broackes-Carter FC, Harris A, Cutting GR. Atypical 5' splice sites cause CFTR exon 9 to be vulnerable to skipping. Am J Hum Genet 2002;71:294-303.

19. Groman JD, Hefferon TW, Casals T, Bassas L, et al. Variation in a repeat sequence determines whether a common variant of the cystic fibrosis transmembrane conductance regulator gene is pathogenic or benign. Am J Hum Genet 2004;74:176-179.

20. Hefferon TW, Groman JD, Yurk CE, Cutting GR. A variable dinucleotide repeat in the CFTR gene contributes to phenotype diversity by forming RNA secondary structures that alter splicing. Proc Natl Acad Sci U S A 2004;101:3504-3509.

21. Audrézet MP, Chen JM, Raguénès Chuzhanova N, et al. Genomic rearrangements in the CFTR gene: extensive allelic heterogeneity and diverse mutational mechanisms. Hum Mutat 2004;23:343-357.

22. Niel F, Martin J, Moal FD, Costes B, et al. Rapid detection of CFTR gene rearrangements impacts on genetic counselling in cystic fibrosis. J Med Genet 2004;41:e118.

23. Bombieri C, Bonizzato A, Castellani C, Assael BM, et al. Frequency of large CFTR gene rearrangements in Italian CF patients. Eur J Hum Genet 2005;13:687-689.

24. Hantash FM, Redman JB, Starn K, Anderson B, et al. Novel and recurrent rearrangements in the CFTR gene: clinical and laboratory implications for cystic fibrosis screening. Hum Genet 2006;119:126-136.

25. Hantash FM, Milunsky A, Wang Z, Anderson B, et al. A large deletion in the CFTR gene in CBAVD. Genet Med 2006;8:93-95.

26. Sousa M, Cremades N, Silva J, Oliveira C, et al. Predictive value of testicular histology in secretory azoospermic subgroups and clinical outcome after microinjection of fresh and frozen-thawed sperm and spermatids. Hum Reprod 2002;17:1800-1810.

27. Fanen P, Ghanem N, Vidaud M, Besmond C, et al. Molecular characterization of cystic fibrosis: 16 novel mutations identified by analysis of the whole cystic fibrosis conductance transmembrane (CFTR) coding regions and splice site mutations. Genomics 1992;13:770-776.

28. Costes B, Fanen P, Goossens M, Ghanem N. A rapid, efficient and sensitive assay for simultaneous analysis of multiple cystic fibrosis mutations. Hum Mutat 1993;2:185191.

29. Maréchal C, Audrézet M, Quéré I, Raguénès $O$, et al. Complete and rapid scanning of the cystic fibrosis transmembrane conductance regulator (CFTR) gene by dena- 


\section{Grangeia et al.}

turing high-performance liquid chromatography (D-HPLC): major implications for genetic counselling. Hum Genet 2001;108:290-298.

30. Dork T, Dworniczak B, Aulehla-Scholz C, Wieczorek D, et al. Distinct spectrum of CFTR gene mutations in congenital absence of the vas deferens. Hum Genet 1997; 100:365-377.

31. Dörk T, Macek M, Mekus F, Tümmler B, et al. Characterization of a novel 21-kb deletion, CFTRdele2,3(21kb), in the CFTR gene: a cystic fibrosis mutation of Slavic origin common in Central and East Europe. Hum Genet 2000;106:59-68.

32. Kanavakis E, Tzetis M, Antoniadi TH, Pistofidis G, et al. Cystic fibrosis mutation screening in CBAVD patients and men with obstructive azoospermia or severe oligozoospermia. Mol Hum Reprod 1998;4:333-337.

33. Casals T, Bassas L, Egozcue S, Ramos MD, et al. Heterogeneity for mutations in the CFTR gene and clinical correlations in patients with congenital absence of the vas deferens. Hum Reprod 2000;15:1476-1483.

34. Bernardino ALF, Lima CE, Zatz M. Analysis of mutations in the cystic fibrosis transmembrane regulator (CFTR) gene in patients with obstructive azoospermia. Genet Mol Biol 2003;26:1-3.

35. Dayangaç D, Erdem H, Yilmaz E, Sahin A, et al. Mutations of the CFTR gene in Turkish patients with congenital bilateral absence of the vas deferens. Hum Reprod 2004;19:1094-1100.

36. Grangeia A, Carvalho F, Fernandes S, Silva J, et al. A novel missense mutation P1290S at exon-20 of the CFTR gene in a Portuguese patient with congenital bilateral absence of the vas deferens. Fertil Steril 2005;83:448-451.

37. Radpour R, Gilani MA, Gourabi H, Dizaj AV, et al. Molecular analysis of the IVS8-T splice variant 5T and M470V exon 10 missense polymorphism in Iranian males with congenital bilateral absence of the vas deferens. Mol Hum Reprod 2006;12:469-473.

38. Dohle GR, Veeze HJ, Overbeek SE, van den Ouweland AMW, et al. The complex relationships between cystic fibrosis and congenital bilateral absence of the vas deferens: clinical, electrophysiological and genetic data. Hum Reprod 1999;14:371374

39. Pradal U, Castellani C, Delmarco A, Mastella G. Nasal potential difference in congenital bilateral absence of the vas deferens. Am J Respir Crit Care Med 1998;158: 896-901.

40. Lissens W, Mahmoud KZ, El-Gindi E, Abdel-Sattar A, et al. Molecular analysis of the cystic fibrosis gene reveals a high frequency of the intron 8 splice variant $5 \mathrm{~T}$ in Egyptian males with congenital bilateral absence of the vas deferens. Mol Hum Reprod 1999;5:10-13.
41. Wang Z, Milunsky J, Yamin M, Maher T, et al. Analysis by mass spectrometry of 100 cystic fibrosis gene mutations in 92 patients with congenital bilateral absence of the vas deferens. Hum Reprod 2002;17:2066-2072.

42. Wu CC, Hsieh-Li HM, Lin YM, Chiang HS. Cystic fibrosis transmembrane conductance regulator gene screening and clinical correlation in Taiwanese males with congenital bilateral absence of the vas deferens. Hum Reprod 2004;19:250-253.

43. Levy EM, Granados P, Rawe V, Olmedo SB, et al. Mutations in CFTR gene and clinical correlation in Argentine patients with congenital bilateral absence of the vas deferens. Medicina (B Aires) 2004;64:213-218.

44. Uzun S, Gokce S, Wagner K. Cystic fibrosis transmembrane conductance regulator gene mutations in infertile males with congenital bilateral absence of the vas deferens. Tohoku J Exp Med 2005;207:279-285.

45. Teng H, Jorissen M, Van Poppel H, Legius E, et al. Increased proportion of exon 9 alternatively spliced CFTR transcripts in vas deferens compared with nasal epithelial cells. Hum Mol Genet 1997;6:85-90.

46. Rave-Harel N, Kerem E, Nissim-Rafinia M, Madjar I, et al. The molecular basis of partial penetrance of splicing mutations in cystic fibrosis. Am J Hum Genet 1997;60: $87-94$

47. Cutting GR. Modifier genetics: cystic fibrosis. Annu Rev Genomics Hum Genet 2005; 6:237-260.

48. Chen JM, Cutler C, Jacques C, Boeuf G, et al. A combined analysis of the cystic fibrosis transmembrane conductance regulator: implications for structure and disease models. Mol Biol Evol 2001;18:1771-1788.

49. Anderson MP, Welsh MJ. Regulation by ATP and ADP of CFTR chloride channels that contain mutant nucleotide-binding domains. Science 1992;257:17011704

50. Carson MR, Travis SM, Welsh MJ. The two nucleotide-binding domains of cystic fibrosis transmembrane conductance regulator (CFTR) have distinct functions in controlling channel activity. J Biol Chem 1995;270:1711-1717.

51. Berger AL, Ikuma M, Hunt JF, Thomas PJ, et al. Mutations that change the position of the putative gamma-phosphate linker in the nucleotide binding domains of CFTR alter channel gating. J Biol Chem 2002;277:2125-2131.

52. Schrijver I, Ramalingam S, Sankaran R, Swanson S, et al. Diagnostic testing by CFTR gene mutation analysis in a large group of Hispanics: novel mutations and assessment of a population-specific mutation spectrum. J Mol Diagn 2005;7: 289-299.

53. Mercier B, Verlingue C, Lissens W, Silber SJ, et al. Is congenital bilateral absence of vas deferens a primary form of cystic fibrosis? Analyses of the CFTR gene in 67 patients. Am J Hum Genet 1995;56:272-277. 